\title{
Pilot Study of Change in Intraabdominal Pressure after Pelvic Organ Prolapse Reconstructive Surgery for Uterovaginal Prolapse
}

\author{
Serdar Aydın ${ }^{1}$, Sinan Yllmaz ${ }^{1}$, Seda Ates ${ }^{1}$, Nezlihan Bademler ${ }^{1}$, and Ayse Filiz Gokmen \\ Karasu $^{1}$ \\ ${ }^{1}$ Bezmialem Vakif University
}

June 15, 2020

\begin{abstract}
Objective: Intra-abdominal pressure (IAP) was defined as the steady state pressure concealed within the abdominal cavity. Sustained increased in IAP has become the focus of attention in many disciplines which is has impact on pulmonary, cardiovascular systems. Pelvic organ prolapse might be the consequences of compensation of abdominal compliance to increased IAP. The aim of this study was to evaluate the effect of laparoscopic sacrocolpopexy in patients with severe uterovaginal prolapse on intraabdominal presurre. Design: Prospective, case control study Setting: Tertiary Urogynecology Unit Population: Women with advanced symptomatic stage [?]3 uterovaginal prolapse. Method: IAPs were measured in 13 women, before and 6 month after laparoscopic sacrocolpopexy and in 13 controls. Main Outcome Measure: Preoperative and postoperative IAP. Results: The mean preoperative IAP of controls $(4.5 \pm 1 \mathrm{mmHg})$ was not significantly different than to women with stage [?]3 uterovaginal prolapse $(\mathrm{p}=0.1)$. The mean postoperative IAP at 6 months control was $8.6+-2.5 \mathrm{mmHg}$ and significantly higher than postprocedure IAP of control group $(4.8+-1.1 \mathrm{mmHg})(\mathrm{p}<0.0001)$. IAP of prolapse group was significantly correlated with gravidy $(\mathrm{r}=0.65, \mathrm{P}<0.01)$ and parity $(\mathrm{r}=0.87, \mathrm{P}<0.001)$. Conclusions: IAP significantly increases after pelvic reconstructive surgery in our pilot study. The association of increased IAP with pelvic organ prolapse and its clinical consequences should be evaluated with large, well designed, with studies
\end{abstract}

\section{Introduction}

Pelvic organ prolapse (POP), defined as the herniation of the pelvic organs to or beyond the vaginal walls, is a common condition. Uterine procidentia is hernia of all three compartments through the vaginal introitus. POP commonly occurs in the elderly and risk increases with age. ${ }^{1}$ Beyond advancing age and vaginal delivery, obesity and in cases where intra-abdominal pressure increases repetitively or continuously such as chronic constipation, chronic obstructive pulmonary disease, or occupations that involve heavy lifting are established risk factors. ${ }^{2-5}$ Anatomic support of the pelvic organs in women is provided by the pelvic floor muscles and ligaments or connective tissue attachments to the pelvis. Excessive caudal movement of pelvic organs occurs with the loss of support impairs the ability of that support to resist caudal forces that include gravity, inertial forces and intra-abdominal pressure. The compliance of these structures, defined as the deformation of the structure divided by the change in force that caused the deformation, describes the pliability or flexibility of pelvic floor and abdominal wall structures.

Intra-abdominal pressure (IAP) is defined as the steady state pressure concealed within the abdominal cavity. IAP, intra-abdominal hypertension (IAH) and abdominal compartment syndrome/pathophysiology have become the focus of attention in many disciplines. ${ }^{6}$ A normal IAP varies from sub-atmospheric values to $7 \mathrm{mmHg}$ in normal weight individuals, with higher baseline levels in morbidly obese patients of about 9 to $14 \mathrm{mmHg} .^{7}$ IAH is defined as a sustained increase in IAP [?] $12 \mathrm{mmHg}$. The adverse physiologic effects of increase in IAP impacts the pulmonary, cardiovascular, renal, splanchnic, musculoskeletal and central nervous systems. ${ }^{8}$ However, little concern has been dedicated to the potential importance of the structure of 
the pelvic floor and its compliance. Abdominal compliance is defined as a measure of the ease of abdominal expansion, which is determined by the elasticity of the abdominal wall and diaphragm. The abdomen may be considered a closed box. This box has rigid structures, i.e. the spine and pelvic bones, with partially flexible sides, i.e. the abdominal wall, diaphragm and pelvic floor. ${ }^{9}$ Levator hiatus is the opening of this closed box to the atmosphere. Pelvic organ prolapse might be the consequence of the compensation of abdominal compliance to chronic increased IAP. We hypothesized that we could disrupt this feasible compensation with relatively stable reconstructive procedures like sacrocolpopexy or obliterative procedures. The aim of this study was to evaluate the effect of pelvic reconstructive surgery in patients with severe uterovaginal prolapse on IAP.

\section{Materials and Methods}

This was a cross-sectional, case-control study comparing preoperative and postoperative IAP in patients advanced symptomatic uterovaginal prolapse stage 3 or 4 according to the Pelvic Organ Prolapse Quantification (POP-Q) system. Each woman in the study group was paired to a woman of the same age ( \pm 3 years) and similar body mass index (BMI) $( \pm 2)$ with POP stage [?] 2; they underwent a diagnostic dilatation and curettage $(\mathrm{D} \& \mathrm{C})$ procedure for postmenopausal bleeding or increased endometrial thickness. These women constituted the control group. Women with previous abdominal or inguinal hernia surgery, previous prolapse surgery, hysterectomy, severe heart disease, lung disease and women who preferred conservative management or uterus-sparing surgery were excluded from the study. Written informed consent was obtained from all patients before enrolment. This study was approved by the institutional review board. Demographic data including age at surgery, parity, menopausal status, BMI, previous surgeries and comorbidities were obtained from patient medical records.

\section{Pressure measurement}

IAP was measured by a single experienced investigator preoperatively before the pelvic organ prolapse reconstruction procedure or $\mathrm{D} \& \mathrm{C}$. Before the induction of any general or spinal anesthesia, a Foley catheter was placed, as is our current standard of care.

IAP measurements were obtained with the patient in the fully supine position without head of bed elevation and at the end of expiration, according to the recommendations by the World Society of the Abdominal Compartment Syndrome (WSACS). ${ }^{7}$ The mid-axillary line at the level of the iliac crest was used as the zero reference point. The Foley catheter was clamped 2 centimeters distal to the port. The pressure transducer system was connected to the pressure monitoring cable tubing was then connected to the Foley catheter via a 16-gauge needle through the port on the catheter tubing. A $50 \mathrm{~mL}$ syringe was attached to the distal stopcock and filled with saline, and the stopcock was turned off toward the patient. Then, $50 \mathrm{cc}$ of sterile saline was instilled into the bladder and the measurement of IAP was performed for 30-60 seconds, after pressure stabilization and the end of respiratory expiration were ensured.10 The second measurement was performed 6 months after prolapse surgery with the same protocol.

\section{Surgical technique}

For the laparoscopic sacrocolpopexy procedure, after vaginal or laparoscopic hysterectomy was performed, the anterior vaginal wall was dissected up to the bladder neck, and the posterior vaginal wall was dissected up to the distal levator ani muscle level. The sacral promontory was identified and the overlying peritoneum was opened up to the vaginal cuff laterally to the rectum and medially to the right uterosacral ligament. A Y-shaped, $15 \mathrm{~cm}$-long, $3 \mathrm{~cm}$-width type 1 polypropylene mesh was transfixed to the anterior and posterior vaginal walls with a 4-6 polyglactin suture in each wall. The free arm of the Y mesh was fixated to the anterior longitudinal ligament of the sacrum with two separate no. 1 polypropylene sutures. The mesh was then peritonealized with absorbable interrupted extracorporeal sutures.

\section{Statistical analysis}

Statistical analysis was performed after normality testing (histogram analysis and/or Kolmogorov-Smirnov test) using IBM SPSS, version 21 (IBM Inc., Armonk, NY). Two sided paired Student's t-tests or Wilcoxon 
signed rank tests were used to compare the IAP values before and after pelvic reconstructive surgery. Student's t-test was used for the comparison of normally distributed variables, while the Mann-Whitney U-test was used for non-parametric variables, and Fisher's exact tests were used to compare categorical variables. Pearson's correlations were used to assess correlations between IAP and patient characteristics such as age, weight, waist circumference and parity. Spearman's correlation was applied in cases where the normality of the data was questionable. AP value of $<0.05$ was considered significant for all tests.

\section{Results}

A total of 52 sets of IAP measurements were conducted in 26 women: 13 in the prolapse group and 13 in the control D\&C group. Thirteen women with stage 3 or utero-vaginal prolapse 4 according to the POP-Q system underwent the sacrocolpopexy procedure. The demographics and IAP measurements of each group are shown in Table 1. As a result of matching, there was no significant difference in age and BMI between both groups. The mean age was $62+-10.7$ years (range 43-76) in the prolapse group and $61.5+-10.1$ years (range 44-75) in control group. The mean BMI was 26.4 +- 2.3 (range 22.6-30) in the prolapse group and $27.9+-2.2$ (range 23-32) in the control group. The waist circumferences were similar between the prolapse $(104.2+-8.9)$ and control (102.5 +- 9.1) groups. The median gravida was significantly higher in the prolapse group (median 4 (range 2-6)) than in the control group (median $3(1-4), \mathrm{p}=0.006$ ). The median parity was $3(2-6)$ in the prolapse group and $2(1-4)$ in the controls $(\mathrm{p}=0.5)$.

The mean preoperative IAP was $5.6+-2.4 \mathrm{~mm} \mathrm{Hg}$ (range $2-11 \mathrm{~mm} \mathrm{Hg}$ ) in the prolapse group. The mean preoperative IAP of controls $(4.5+-1 \mathrm{~mm} \mathrm{Hg}$ (range $3-6 \mathrm{~mm} \mathrm{Hg}$ ) was not significantly different than in women with stage [?]3 uterovaginal prolapse $(\mathrm{p}=0.1)$. Preoperative IAP of $4(53.8 \%)$ women with prolapse was over $7 \mathrm{mmHg}(\mathrm{p}=0.03)$. The mean postoperative IAP at the 6 month control examination was $8.6+-2.5$ $\mathrm{mmHg}$ (range 6-14 $\mathrm{mmHg}$ ) and significantly higher than the postprocedure IAP of the control group (4.8 +$1.1 \mathrm{mmHg}$ (range $3-7 \mathrm{mmHg})(\mathrm{p}<0.0001)$. Figure 1 shows the intra-abdominal pressure variations pre- and postoperatively. The mean IAP was significantly higher at 6 months after pelvic reconstructive surgery than before surgery $(\mathrm{p}=0.001)$. The alteration of IAP in the control group was statistically insignificant $(\mathrm{p}=0.3)$. The IAP of seven $(53.8 \%)$ patients was over $7 \mathrm{mmHg}(\mathrm{p}=0.03)$, and 2 of $13(15.3 \%)$ had IAH $(\mathrm{p}=0.1)$.

In the correlation analysis of preoperative and postoperative IAP with age, BMI, weight, height, gravidy and parity of women, postoperative IAP in the prolapse group was significantly correlated with gravidy $(\mathrm{r}$ $=0.65, \mathrm{P}<0.01)$ and parity $(\mathrm{r}=0.87, \mathrm{P}<0.001)$. Age, BMI, weight, height and waist circumference were not correlated with either preoperative or postoperative IAP measurements.

\section{Discussion}

In this prospective controlled study, we evaluated the effect of prolapse surgery with mesh on IAP alterations. We observed that the postoperative IAPs after uterovaginal prolapse surgery were elevated in comparison to the preoperative values. The question of this study was raised at an urogynecology congress at which an experienced anesthesiologist hypothesized that the reentrance of pelvic contents and fixation of the vaginal wall with rigid materials may have long-term health consequences. There is no answer to this question to date, according to the current evidence. There have been no studies on the consequences of gynecologic procedures on IAP measurements or the effect of IAP changes on short- or long-term outcomes. Although there have been limited studies relevant to the effect of pregnancy and cesarean delivery on IAP, in case reports after gynecologic emergencies, there is a lack of data after gynecologic surgery, especially urogynecologic procedures commonly dealing with the elderly and frail population. ${ }^{11-13}$ With increasing age, cardiovascular and respiratory comorbidities may complicate pelvic organ prolapse in those older patients who are more susceptible to IAP alterations and need to be more cautions. ${ }^{14}$

Midterm postoperative IAPs after laparoscopic sacrocolpopexy were elevated in comparison to the preoperative values. This may be explained by a variety of mechanisms. The fixation of relatively rigid and non-absorbable polypropylene mesh diminishes vaginal wall distension and movement properties. Rubod et al. reported that vaginal tissue from prolapse patients exhibits larger deformations and behaves in a hyperelastic manner with increased compliance. ${ }^{15}$ 
Although the aim of the mesh is to strengthen the impaired vaginal wall, mimicking autologous tissue without reducing its compliance, the stiffness of the material, shrinkage and new tissue formation are associated with poor compliance. ${ }^{16}$ The usage of synthetic materials could be related to the increase in IAP secondary to a disruption or compensation mechanism.

Another probable mechanism may be related to an increase in the pelvic contents after prolapse surgery, due to the introduction of the bladder, bowel and uterus into the pelvic cavity, leading to a subsequent increase in IAP. Similar mechanisms can also account for increased IAP or IAH after abdominal wall hernia repair. In a few studies examining the association between hernia repair and intraabdominal pressure, ventral hernia repair can be associated with perioperative intra-abdominal hypertension (IAH), respiratory dysfunction and complications.${ }^{17-19}$ In a study on large incisional hernias, $87 \%$ of patients showed a mean increase in IAP of $2.7 \mathrm{mmHg}$ after surgery; about $9 \%$ saw no change in pressure. ${ }^{20} \mathrm{In}$ a cadaveric model, IAP increased by about $4.6 \mathrm{~mm}$ with increasing volume in the pelvic cavity. ${ }^{21}$ The rise in IAP during abdominal surgery observed in our study can be explained by the stretch of the abdominal wall following hernia repair [19]. Also, IAP can increase under high-tension abdominal wall closure and can be considered the cause of complications such as recurrences and respiratory insufficiency and post-operative pneumonia, but there is a lack of long-term data about these alterations in pressure. ${ }^{22,} 23$

We found a strong correlation with parity and increased IAP in our study. IAP may increase postoperatively due to abdominal cavity characteristics. The abdominal wall will modify its constitutional properties to maintain them as close as possible to normal functioning under the alterations in IAP. The high IAP after pelvic reconstructive surgery in women with high parity can be explained by weak compliance of the abdominal musculoaponeurotic system after repeated pregnancies. ${ }^{24}$ Although a BMI and IAP correlation was not found in our study population, we matched the control group with similar BMI to eliminate the BMI limitation.

\section{Strengths and limitations}

To the best of our knowledge, our study is the first to evaluate the association between reconstructive surgery of severe uterovaginal prolapse and IAP. The second strength of the study is the homogenous population and that all procedures were standard laparoscopic sacrocolpopexy procedures in order to eliminate the effect of abdominal incision. A major limitation of the study is the small study population and lack of sample size calculation to prevents us from drawing clear conclusions. Secondly, we did not perform early postoperative IAP measurements, as pain and respiratory changes might influence the measured IAP. Another limitation is the lack of inter-observer variability since all the measurements were performed by the same experienced investigator.

\section{Conclusion}

POP has been accepted as a quality of life problem rather than a comorbidity. The long-term outcome studies of prolapse surgery are often focused on recurrence and complications. To date, little is known about survival or the long-term health consequences of POP after reconstructive pelvic surgery. The survival rates after pelvic reconstructive surgeries assessed by inference from studies on patients aged 65 years or older undergoing elective general surgery were similar to those who did not undergo surgery. ${ }^{25}$ IAP was significantly increased after pelvic reconstructive surgery in our pilot study. Increased IAP after reconstructive pelvic surgery may be associated with long-term unfavorable health consequences. The association between increased IAP with POP and its clinical consequences should be evaluated in large, well-designed studies.

\section{Disclosure of interests}

The authors report no conflicts of interest. The authors alone are responsible for the content and writing of this paper

\section{Contribution to authorship}

SA and SY conceived and designed the study. AFGK, SA and SY formulated the research questions. NB and 
SA were responsible for data management and statistical analysis. AFGK, SA and SA analyzed the data. SA and SY drafted the paper, which was revised and approved by all authors. AFGK and NB contributed by revising the manuscript and providing important input.

\section{Details of ethics approval}

The Ethical Review Board at the Bezmialem Vakif University of Istanbul (2018-6590) approved the study. Date of approval 04 April 2018.

\section{Funding}

None

\section{References}

1. Swift S, Woodman P, O'Boyle A, et al. Pelvic Organ Support Study (POSST): the distribution, clinical definition, and epidemiologic condition of pelvic organ support defects. Am J Obstet Gynecol. 2005;192(3):795-806. doi:10.1016/j.ajog.2004.10.602

2. Weber AM, Walters MD, Ballard LA, et al. Posterior vaginal prolapse and bowel function. Am J Obstet Gynecol 1998; 179:1446.

3. Spence-Jones C, Kamm MA, Henry MM, Hudson CN. Bowel dysfunction: a pathogenic factor in uterovaginal prolapse and urinary stress incontinence. Br J Obstet Gynaecol 1994; 101:147.

4. Woodman PJ, Swift SE, O'Boyle AL, et al. Prevalence of severe pelvic organ prolapse in relation to job description and socioeconomic status: a multicenter cross-sectional study. Int Urogynecol J Pelvic Floor Dysfunct 2006; 17:340.

5. Vergeldt TF, Weemhoff M, IntHout J, Kluivers KB. Risk factors for pelvic organ prolapse and its recurrence: a systematic review. Int Urogynecol J 2015; 26:1559.

6. Malbrain ML, Cheatham ML, Kirkpatrick A et al.: Results from the International Conference of Experts on Intra-abdominal Hypertension and Abdominal Compartment Syndrome. I. Definitions. Intensive Care Med 2006; 32: 1722-1732.

7. Kirkpatrick AW, Roberts DJ, De Waele J, et al. Intra-abdominal hypertension and the abdominal compartment syndrome: updated consensus definitions and clinical practice guidelines from the World Society of the Abdominal Compartment Syndrome. Intensive Care Med. 2013;39(7):1190-1206. doi:10.1007/s00134-013-2906-z

8. Cheatham ML, Safcsak K, Sugrue M. Long-term implications of intra-abdominal hypertension and abdominal compartment syndrome: physical, mental, and financial. Am Surg. 2011;77 Suppl 1:S78S82.

9. Malbrain ML, De laet IE. Intra-abdominal hypertension: evolving concepts. Clin Chest Med. 2009;30(1):45-viii. doi:10.1016/j.ccm.2008.09.003

10. Lee RK. Intra-abdominal hypertension and abdominal compartment syndrome: a comprehensive overview. Crit Care Nurse. 2012;32(1):19-31. Review.

11. Sugerman HJ. Hypothesis: preeclampsia is a venous disease secondary to an increased intra-abdominal pressure. Med Hypotheses. 2011;77(5):841-849. doi:10.1016/j.mehy.2011.07.051

12. Fuchs F, Bruyere M, Senat MV, Purenne E, Benhamou D, Fernandez H. Are standard intra-abdominal pressure values different during pregnancy?. PLoS One. 2013;8(10):e77324.

13. Sanda RB, Aziz R, Bhutto A, Seliem SI. Abdominal Compartment Syndrome complicating massive hemorrhage from an unusual presentation of ruptured ectopic pregnancy. Ann Afr Med. 2011;10(3):252-255. doi:10.4103/1596-3519.84700

14. Papavramidis TS, Lazou TP, Cheva A, et al. Chronically increased intra-abdominal pressure: validating a model. Obes Surg 2010;20:900-5.

15. Rubod C, Boukerrou M, Brieu M, Jean-Charles C, Dubois P, Cosson M. Biomechanical properties of vaginal tissue: preliminary results. Int Urogynecol J Pelvic Floor Dysfunct. 2008;19(6):811-816.

16. Todros S, Pavan PG, Pachera P, Natali AN. Synthetic surgical meshes used in abdominal wall surgery: Part II-Biomechanical aspects. J Biomed Mater Res B Appl Biomater. 2017;105(4):892- 
903. doi:10.1002/jbm.b.33584

17. Gaidukov KM, Raibuzhis EN, Hussain A, et al. Effect of intra-abdominal pressure on respiratory function in patients undergoing ventral hernia repair. World J Crit Care Med. 2013;2(2):9-16. Published 2013 May 4. doi:10.5492/wjccm.v2.i2.9

18. Papavramidis TS, Kotidis E, Ioannidis K, et al. Intra-abdominal pressure changes after inguinal hernia repair. Am Surg. 2012;78(1):E12-E14.

19. De Santis L, Frigo F, Bruttocao A, Terranova O. Pathophysiology of giant incisional hernias with loss of abdominal wall substance. Acta Biomed. 2003;74 Suppl 2:34-37

20. Angelici AM, Perotti B, Dezzi C, et al. Measurement of intra-abdominal pressure in large incisional hernia repair to prevent abdominal compartmental syndrome. G Chir. 2016;37(1):31-36. doi:10.11138/gchir/2016.37.1.031

21. Kohler D, Sellei RM, Sop A, et al. Effects of pelvic volume changes on retroperitoneal and intraabdominal pressure in the injured pelvic ring: a cadaveric model. J Trauma. 2011;71(3):585-590. doi:10.1097/TA.0b013e318224cd62

22. Venclauskas L, Maleckas A, Kiudelis M. One-year follow-up after incisional hernia treatment: results of a prospective randomized study. Hernia. 2010;14(6):575-582. doi:10.1007/s10029-010-0686-8

23. Bezzi M, Nasti AG, Simonelli I, Bosco MR, Leonetti G, Angelici AM. Large incisional hernia in the elderly: which kind of treatment? Acta Biomed. 2005;76( suppl):21-23.

24. Papavramidis TS, Lazou TP, Cheva A, et al. Chronically increased intra-abdominal pressure: validating a model. Obes Surg 2010;20:900-5.

25. Gerten KA, Richter HE. Pelvic floor surgery in the older woman. Clin Obstet Gynecol. 2007;50(3):826843. doi:10.1097/GRF.0b013e3180de4702

\begin{tabular}{llll}
\hline & Prolapse Group & Control Group & P value \\
\hline Age, mean $\pm \mathrm{SD}^{*}$, years & $62 \pm 10.7$ & $61.9 \pm 10.1$ & 0.9 \\
BMI, mean $\pm \mathrm{SD} *$ & $26.4 \pm 2.3$ & $27.9 \pm 2.2$ & 0.7 \\
Waist Circumference mean $\pm \mathrm{SD}{ }^{*}, \mathrm{~cm}$ & $104.2 \pm 8.9$ & $102.5 \pm 9.1$ & 0.6 \\
Gravidy, median (range) + & $4(2-6)$ & $3(1-4)$ & $\mathbf{0 . 0 0 6}$ \\
Parity, median (range) + & $3(2-6)$ & $2(1-4)$ & 0.05 \\
Preoperative IAP, mean $\pm \mathrm{SD}+, \mathrm{mmHg}$ & $5.6 \pm 2.4$ & $4.5 \pm 1$ & 0.1 \\
Preoperative IAP $>7 \mathrm{mmHg} \mathrm{n}(\%)++$ & - & $4(30.8)$ & $\mathbf{0 . 0 3}$ \\
Postoperative IAP mean $\pm \mathrm{SD}+, \mathrm{mmHg}$ & $8.6 \pm 2.5$ & $4.8 \pm 1.1$ & $<\mathbf{0 . 0 0 0 1}$ \\
Postoperative IAP $>7 \mathrm{mmHg}, \mathrm{n}(\%)++$ & - & $7(53.8)$ & $\mathbf{0 . 0 0 2}$ \\
Postoperative IAP $>12 \mathrm{mmHg}, \mathrm{n}(\%)++$ & - & $2(15.4)$ & 0.1 \\
\hline
\end{tabular}

Table 1: Patient characteristic, demographic data and intraabdominal pressure.

* Student t test.

+ Mann-Whitney U test

++ Fisher exact test.

BMI; Body mass index, IAP; Intraabdominal Pressure. Bolding indicates statistical significance 


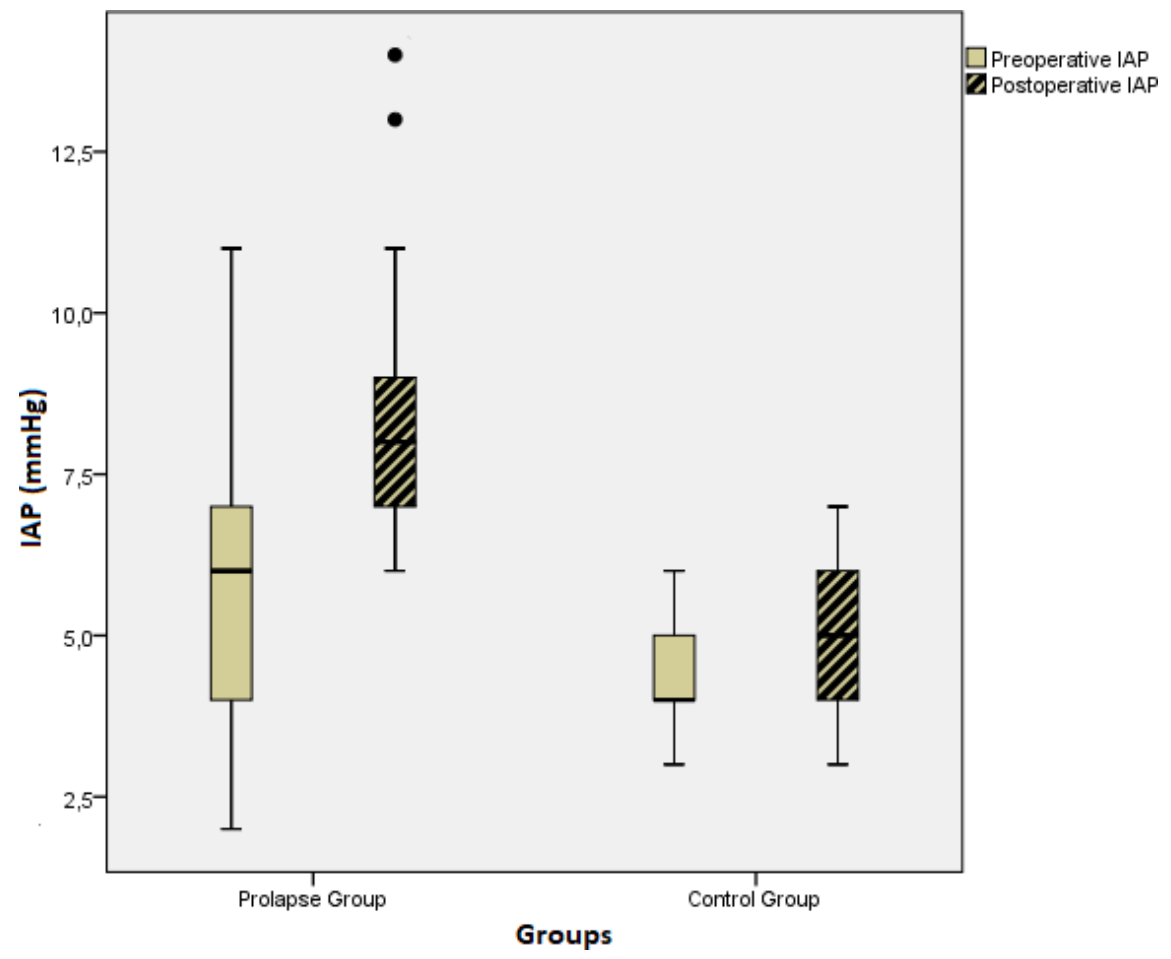

